\title{
Analysis on unbalance protection of SCB
}

\author{
Xiao-ping Xiong ${ }^{1, \mathrm{a}}$, jing-jie $\mathrm{Hu}^{2, \mathrm{~b}}$ and Qiang $\mathrm{Fu}^{3, \mathrm{c}}$ \\ ${ }^{1}$ College of Electric Engineering, Guangxi University, \\ Nanning 530004, Guangxi Zhuang Autonomous Region, China \\ 2, 3 Anyang power supply Bureau, \\ Anyang 455002, Henan Province, China \\ axxp001@sina.com, bdadani2384@hotmail.com, cayfuqiang001@163.com
}

Keywords: unbalance protection; safety; SCB; PSCAD

\begin{abstract}
Shunt capacitor is a main measure to reactive power compensation of power system, which has the advantages of flexibility and economy. In order to guarantee the safety of shunt capacitor, the methods for protecting against over-voltage, under-voltage, over-current and unbalance in circuits according to the different operation modes are used. This paper in detail introduces unbalance protection ways under different connection modes of capacitor group. It is analyzed and calculated that the unbalanced current and voltage with the effects of fault capacitor units, components and fuses on capacitor bank as well through a case of unbalance computation of shunt capacitor banks (SCB) of ungrounded single star with external fuse. It is indicated by PSCAD simulation analysis that the result of theory calculation is the same as the simulation, which provides theory basis of setting the tripping point and alarm point of relay protection.
\end{abstract}

\section{Introduction}

In order to provide the reliable method for stabling voltage, more and more shunt capacitors are used for the power system under the continuous enlargement of the scope of power grid. Shunt capacitor is a kind of important reactive power compensation device in power system, which has the characteristics of simple, economy, convenient and flexible. However, the explosion event of capacitors are occasionally presented, which is caused by overvoltage in operation, illogical configuration settings of device and protection and so on, besides the quality of production itself. It is important that reliability and mutual coordination of the shunt capacitors because of the direct effect of the damage of them on the power quality of substation area. It will further cause the serious accident when the fault occurs in capacitors bank. Therefore, when the protection clears fault in capacitors or components, the unbalance condition of capacitor interior should be alarmed and warned [1-5]. This paper analyzes the common connection modes of shunt capacitor banks (SCB) and their features in brief. The unbalance protection as one of primary relay protection methods is studied and simulated, which provides reference advice on setting the tripping point and alarm point of relay protection. When the fault is occurs, the identification of faulty phase will decrease the repairing time of SCB.

\section{Connections of SCB}

The SCB of high voltage substation is commonly used by the following four connection modes. Fuse and relay protections are two determining aspects of the configuration of capacitor banks. Fig. 1 shows the most common star-connected capacitor bank configuration. If the neutral point grounding type is inrush current, the harmonics can provide a low resistance channel ground. And the recovery voltage of the switching device can also be reduced. If the neutral point grounding type is isolated, it's required that the neutral point must be cut off from live equipment. 


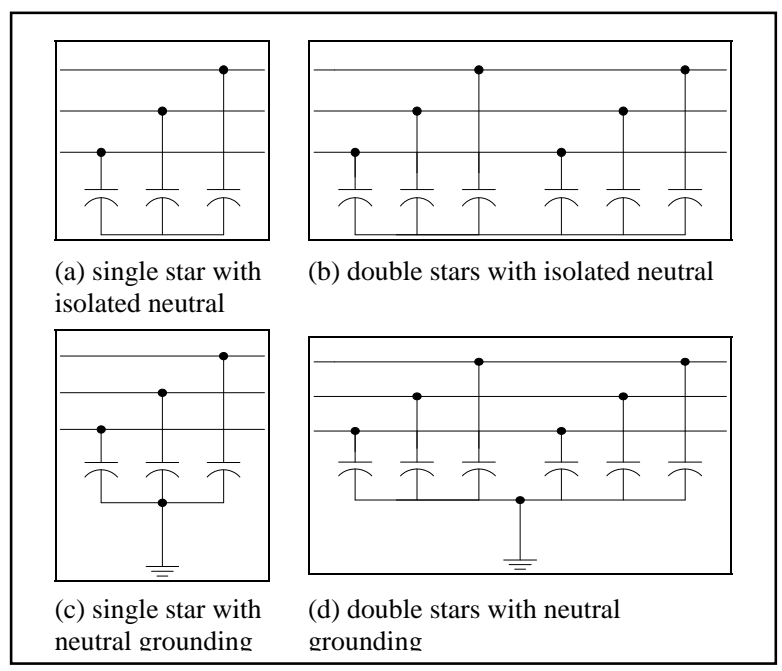

Fig. 1 The four connection modes of SCB

\section{Selection of fuse}

Shunt capacitor is divided into three different types by the fuse, the internal fuse, the external fuse and the no fuse. The fuse protection and the relay protection are two different protection types of shunt capacitor. Under normal circumstances, fuse protection is regarded as the main protection and relay protection is regarded as the backup protection.

Because of the different structures and operation principles of internal fuse capacitor and external fuse capacitor, the mixed use of them is one of the problems often encountered in the protection of shunt capacitor. Therefore, the mixed use of them will not only increase the maintenance work, reduce the reliability of the device, but probably not play a role in protection.

\section{Unbalance protection}

Among the various protection modes of shunt capacitor, over-voltage protection, under-voltage protection, over-current protection and unbalance protection are usually used. Fig. 2 shows a protection scheme for SCB. Over voltage and under voltage protections are mainly used for system failure caused by over-voltage and under-voltage. Current protection is mainly used for system voltage drop caused by short circuit between phases or single phase short circuit. Usually, some external protection devices are used, such as fuses, circuit breakers, line switches, etc. Unbalance protection is mainly used for internal failure of the capacitor banks, such as failure of internal component, failure of internal fuses, components of no fuse capacitor group, failure between poles, failure of capacitor unit wiring, arc flashover within the capacitor bank, and over-voltage due to the malfunctioning component or fuse action[6]. Thus, the unbalance protection is the important part of the internal protections of SCB. The specific protective measures are determined by the selection of fuse, the size of capacitor banks, the choice of grounding, and the insulation of current or voltage transformer.

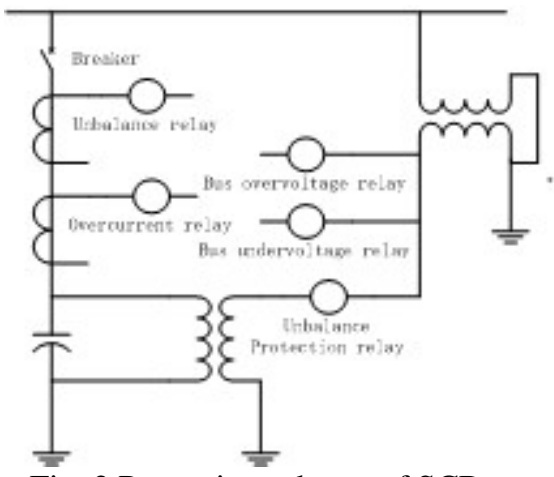

Fig. 2 Protection scheme of SCB 
1. Unbalance Protection of SCB with Ungrounded Single Star. The unbalance protection of SCB with neutral point ungrounded single star has commonly two connection modes. One scheme is not sensitive to system voltage, so its sensitivity is significantly higher than another scheme.

2. Unbalance Protection of SCB with Grounded Single Star. Fig. 3 shows unbalance protection method of single star capacitor bank. The method is set for the differential voltage of three-phase capacitor bank. Its sensitivity is relatively high. And it is usually used in high-voltage capacitor bank composed of large-capacity capacitor units. Current transformers and $\mathrm{H}$ bridge wiring can also be used to build the unbalance current protection program.

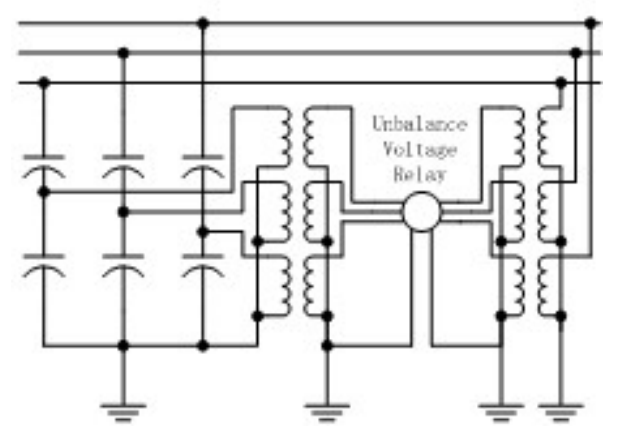

Fig. 3 Protection scheme of SCB with grounded single star

3. Unbalance Protection of SCB with Ungrounded Double Stars. Unbalance protection of SCB with ungrounded double stars is as follows in fig. 4.

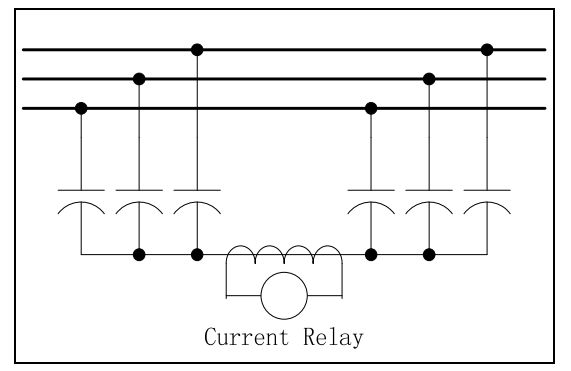

(a)unbalance protection of neutral point current

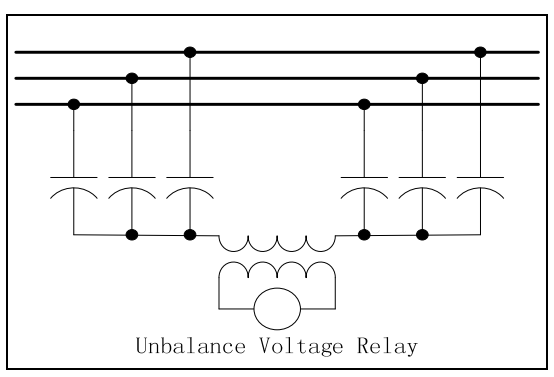

(b)unbalance of neutral isolation

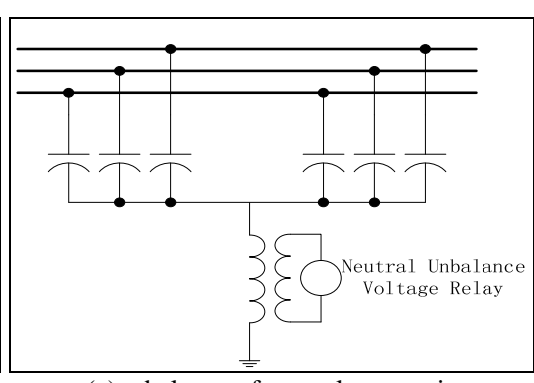

(c)unbalance of neutral connection

Fig. 4 Protection scheme of SCB with ungrounded double stars

Fig. (a) and fig. (b) are two ways to avoid the impact of the system voltage imbalance. When they are in balance, the third harmonic currents or harmonic voltages will also not be affected. The neutral points of the capacitor' two parts in fig. (c) are connected together, voltage transformer is used to measure the voltage of neutral point, but this relay should be provided with filters.

4. Unbalance Protection of SCB with Grounded Double Stars. As shown in figure 5, the star neutral point of the double stars capacitor banks are connected to the same point and grounded. The secondary side of the current transformer is cross-connected with an overcurrent relay. So the relay can react to the outside influence of capacitor.

\section{Unbalance calculation}

In order to make full use of protection relay, it's important to calculate the effect of capacitor units, components and fuses on capacitor banks. So as to determine the set of relay protection point and provide a theoretical basis for the relay setting calculation at the same time. The calculations of the equalities and the equations are determined by the configuration of capacitor banks, the operating parameters of system and the protection methods. According to the parameters calculated by the equalities and the equations, the tripping point of relay can be determined. So these calculations are an important part to measure not only protective systems, but also the sensitivity and reliability of them.

It is simulated by PSCAD in this test. As it's shown in figure 6, SCB with external fuse and single star ungrounded is taken for example. The bus voltage is $35 \mathrm{kV}$, and each phase is 4 series and 4 parallel connected. 
It's assumed that the number of SCB in series with $S$, each SCB having $P$ units and the number of fault capacitor with $N$, and the rate voltage of each capacitor is $U_{0}$. On initial condition, the capacitance of every capacitor unit is one, and the capacitance of each parallel group in series group is $C_{g}$.

$$
C_{g}=\frac{P-N}{P}
$$

The capacitance of fault phase in every capacitor unit is $C_{s}$.

$$
C_{s}=\frac{S \times C_{g}}{C_{g}(S-1)+1}
$$

The neutral point voltage is $V_{n}$

$$
V_{n}=\frac{3}{2+C_{s}}-1
$$

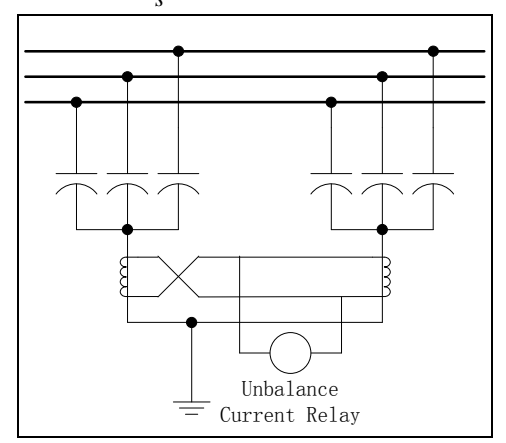

Fig. 5 Protection scheme of SCB with grounded double stars

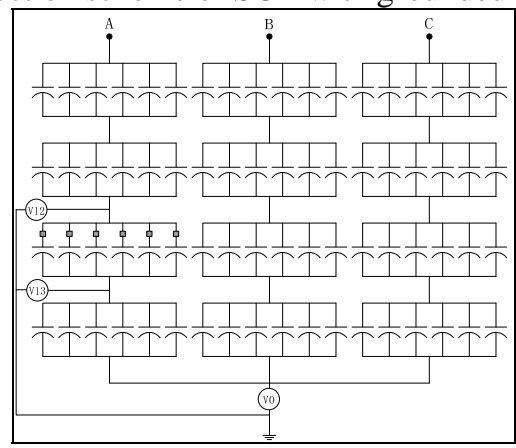

Fig. 6 Protection scheme of SCB with ungrounded single stars

From the Eq. (1), (2) and (3), it is calculated the percentage value of the neutral point voltage. Then the voltage of fault SCB is computed according to the measurement value of V12 and V13.

The algorithm of unbalance voltage and current is reliable and the result indicates the primary data are successfully transformed into secondary data within the allowance range from the table I.

In this example, when a capacitor unit fails, the voltage of other tags capacitor in failure is higher than $1.1 U_{0}$, which is not allowed due to probably resulting in the capacitor breakdown and may further expanding the fault. So when choosing the parallel units of shunt capacitor in high voltage, the number of capacitor unit in parallel should be at least 10 if allowed capacitor banks continuing to run after the resection of a failed capacitor unit. And connection mode, protection mode, the serial number of segments and the allowance multiple times of overvoltage should also be considered. However, if too many capacitor units are in parallel with capacitor banks, the capacitor bank with fuse may not only generate substantial current which may melt the fuse, but also bring difficulty in measuring the relay sensitivity. Therefore, limitation to a maximum number of parallel units is an important aspect of ensuring the safe operation of the capacitor bank. Unbalance protection and other types of protection should also be complement to each other under constraint of the action time of unbalance protection with $0.1 \mathrm{~s} \sim 0.2 \mathrm{~s}$. 


\section{Determination of the fault phase}

SCB played an important role in the reactive power compensation and voltage regulation, so in the repair of them, the interruption time should be as short as possible. The repair time includes the time to identify the point of failure and time to restore the capacitor bank. If we can find the phase of defective unit, it will greatly reduce the scope of search for the point of failure and the time to identify trouble spots.

It is established that an ungrounded SCB of single-star model in PSCAD. Bus voltage is set to $35 \mathrm{kV}$, the number of shunt capacitor in series is 4 , the unit number of per phase shunt capacitor is 4 , the value of each capacitor unit is $37.7 \mu \mathrm{F}$. A1B0C0 means phase A containing a defective unit or component, it is taken the positive sequence voltage $\mathrm{VA}(\mathrm{V} 11)$ of phase $\mathrm{A}$ as a reference. Then the angle between the neutral point $\mathrm{Vn}\left(\mathrm{V}_{0}\right)$ and VA is $180^{\circ}$, as shown in fig. 7 . When the B-phase contains a fault, it will result in $60^{\circ}$ between neutral point Vn and VA. When the C-phase contains a fault, it will result in $-60^{\circ}$ between neutral point $\mathrm{Vn}$ and VA.

Table 2 Comparison and error of calculation and simulation

\begin{tabular}{|l|l|l|l|l|l|l|}
\hline $\begin{array}{l}\text { Number } \\
\text { of fault } \\
\text { units }\end{array}$ & $\begin{array}{l}\mathrm{V} 12 \\
(\mathrm{kV})\end{array}$ & $\begin{array}{l}\mathrm{V} 13 \\
(\mathrm{kV})\end{array}$ & $\begin{array}{l}\text { Voltage of labeled } \\
\text { capacitor in fault } \\
\text { phase }(\mathrm{kV})\end{array}$ & $\begin{array}{l}\text { Voltage of } \\
\text { neutral point } \\
\text { V0(kV) }\end{array}$ & $\begin{array}{l}\text { Calculation of } \\
\text { neutral } \\
\text { voltage } \\
\text { (unit) }\end{array}$ & error \\
\hline 0 & 7.24055 & 3.62028 & 3.62027 & 0.00000 & 0.00000 & $0.000 \%$ \\
\hline 1 & 7.46667 & 3.27561 & 4.19106 & 0.23363 & 0.01613 & $0.021 \%$ \\
\hline 2 & 7.79271 & 2.78781 & 5.00490 & 0.55706 & 0.03846 & $0.021 \%$ \\
\hline 3 & 8.27368 & 2.06871 & 6.20497 & 1.03447 & 0.07143 & $0.008 \%$ \\
\hline 4 & 9.04995 & 0.90437 & 8.14558 & 1.81016 & 0.12500 & $0.001 \%$ \\
\hline 5 & 10.53100 & 1.31641 & 9.21459 & 3.29120 & 0.22727 & $0.003 \%$ \\
\hline 6 & 14.48113 & 7.24078 & - & 7.24078 & 0.50000 & $0.003 \%$ \\
\hline
\end{tabular}

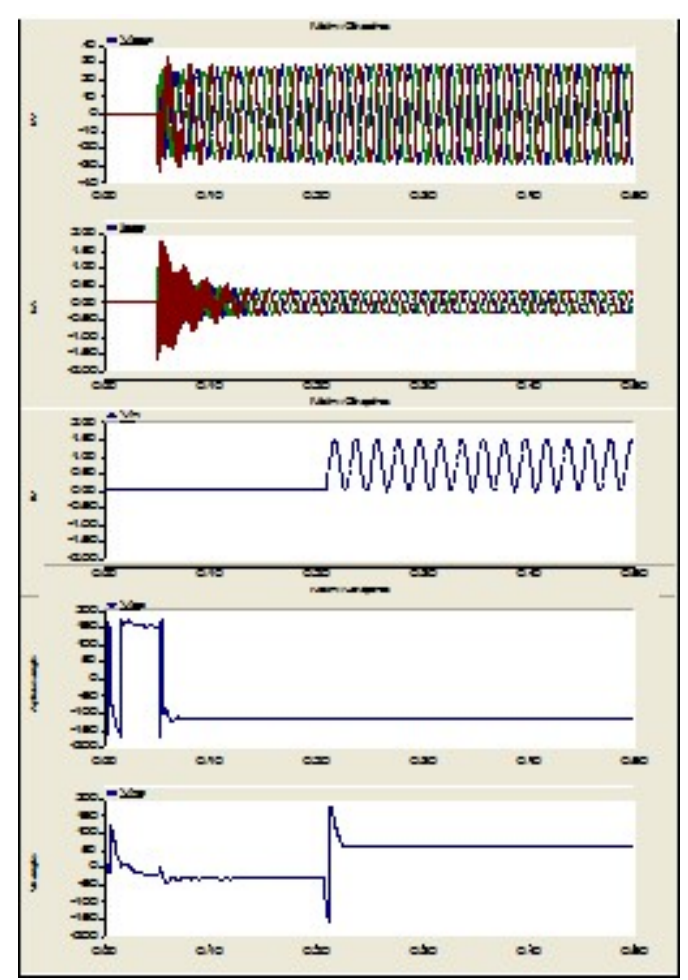

Fig. 7 One fault in phase $\mathrm{A}$

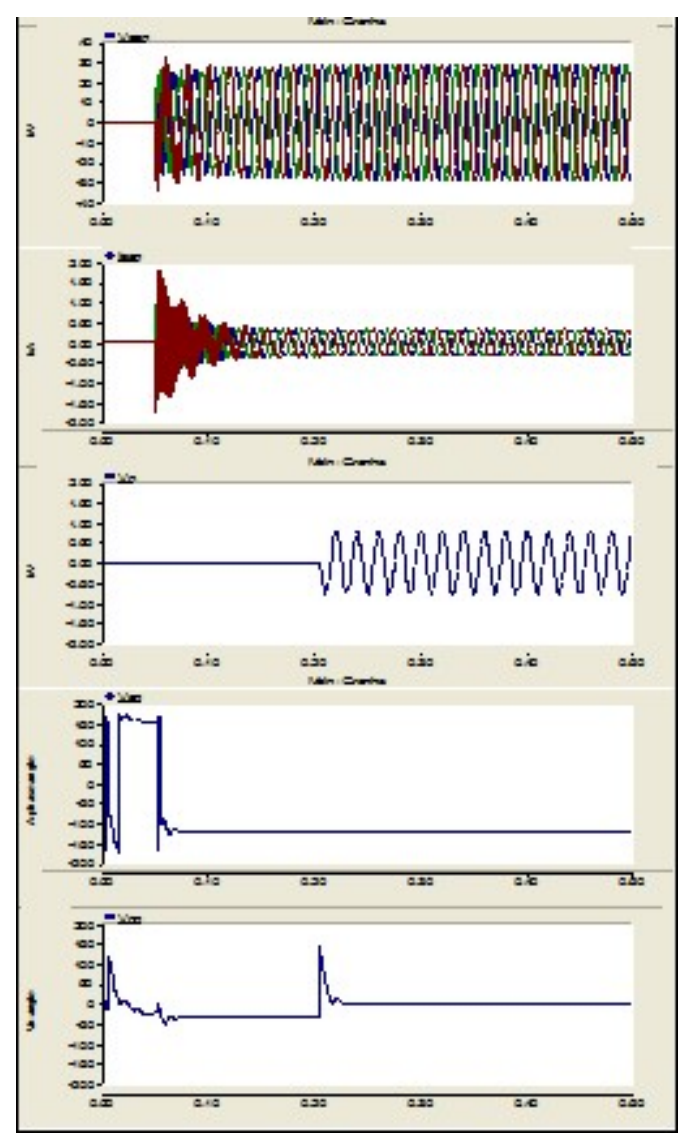

Fig. 8 One fault in each phase A and B

When phase $A$ and phase $B$ each contains a fault, the angle between the neutral point voltage $\mathrm{Vn}$ and VA is $120^{\circ}$. It is showed in fig. 8. When phase $\mathrm{A}$ and phase $\mathrm{C}$ each contains a fault, the angle 
between the neutral point voltage $\mathrm{Vn}$ and VA is $-120^{\circ}$. When both phase $\mathrm{B}$ and phase $\mathrm{C}$ contain a fault, the angle between the neutral point voltage Vn and VA is 0 . When each phase A, B, C contain a fault, the imbalance of neutral point voltage will be eliminated and back to equilibrium point.

As there are many factors can lead the system to an imbalance situation, so in order to verify security and reliability of the program, it is allowed the existence of the error of $\pm 10^{\circ}$. When there is anyone phase fault in capacitor bank, the phase relationship between imbalance voltage of neutral point and the positive sequence voltage is shown in fig. 9.

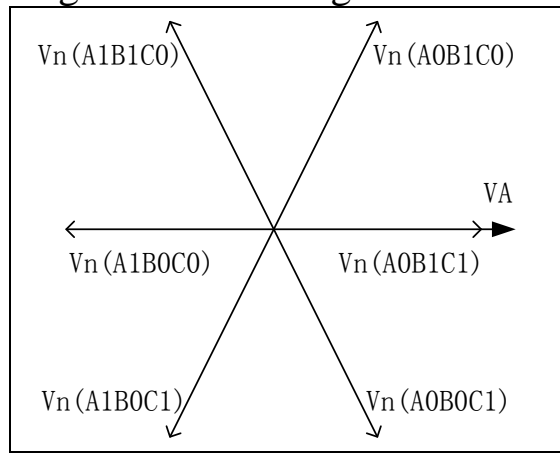

Fig. 9 The phase relation of neutral unbalance voltage and positive sequence voltage

This approach also applies in the form of double stars as well as other types of capacitors connected group, we can greatly shortened the time to determine the failure point if we use this method.

\section{Summary}

When we design SCB, the wiring of capacitor bank and fuse selection should be taken into full account because of affecting the setting of protection. If selected unwisely, it will be a waste of resources, achieve no protective effect and may even cause an accident.

If error is taken into account, the fault current and voltage algorithms are consistent with the result of simulation, which are also reliable. Unbalance protection is an important part of relay protection of SCB. Computational analysis of unbalance protection provides a theoretical basis for the setting of relay tripping points and alarm points.

Fault phase detection method is also suitable for other connection types of capacitor banks. This method greatly reduces the repair time in maintaining SCB and has practical significance in maintenance work.

\section{References}

[1] Gao Y Y and Gao L G, Research on the shunt capacitor protection in power distribution, China rural water and hydropower, 10 (2007) 117-118.

[2] Han X W, Several problems in the design of the fuse structure of the power capacitor protection model, Electrotechnical Journal, 2 (1992) 19-20.

[3] Bishop M, Day T and Chaudhary A, A primer on capacitor bank protection, IEEE Transactions on Power Delivery, 37 (2001) 1174-1179.

[4] Tian Q, Yuan M H and Wang Z P, Configuration, setting and application of capacitor protection in UHV pilot project, High Voltage Apparatus, 3 (2010) 68-71.

[5] Ding Z J , Qu Z F, Xu S G and Wang H, New shunt capacitors protection configuration implemented design, Electrical Measurement \& Instrumentation, 48 (2011) 49-52.

[6] Horton R, T Warren T, Fender K, Harry S and Gross C A, Unbalance protection of fuseless, split-wye, grounded, shunt capacitor banks, IEEE Transactions on Power Delivery, 17(2002) 698-701. 